\title{
The pattern and magnitude of rotational piezomagnetic anomalies along the dip-slip Mosha fault, Northern Tehran, Iran
}

\author{
M. Mokhtari ${ }^{1}$ and S. Kiarasi ${ }^{2}$ \\ ${ }^{1}$ International Institute of Earthquake Engineering and Seismology, No.26, Dibajie Shomali, Tehran, Iran \\ ${ }^{2}$ Azad University, Ponk, Tehran, Iran
}

Received: 21 July 2008 - Revised: 7 October 2008 - Accepted: 8 October 2008 - Published: 28 November 2008

\begin{abstract}
Several active faults affect the Central Alborz, Northern Iran. The main active faults in the region are the North Tehran and the Mosha faults and their westward continuation, the Taleghan fault. Among these the Mosha fault is the most active fault in Central Alborz. The piezomagnetic field has been inspired by many investigations in the earthquake prediction studies and has been reported in the literature. Several theories proposed for relating piezomagnetic changes into magnetic signals accompanying earthquakes in the vicinity of a fault. In this study based on an analytical solution for the elementary piezomagnetic potentials due to an inclined rectangular fault within a semi-infinite elastic medium a computer program has been developed. This program has been used for evaluation of the rotational piezomagnetic changes along the Mosha fault using different earthquakes with specific magnitudes and at the presence of three different geomagnetic main fields. The magnetic data acquired recently at the study area has been used during the modeling. The first result that can be inferred from this study is that rotational piezomagnetic anomalies are completely localized at the fault tips. So for detection of this anomaly the magnetic instruments should be installed at these locations. The other important element which has been investigated was the amount of slip along the fault, which shows anomaly intensification by increase in the amount of slip.
\end{abstract}

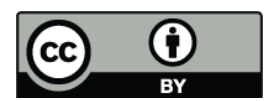

Correspondence to: M. Mokhtari (mokhtari@iiees.ac.ir)

\section{Introduction}

The Mosha Fault, which was first identified by Dellenbach (1964), is the most prominent structure in the southern part of the Central Alborz (Fig. 1). It is an extensive active fault along which Precambrian, Paleozoic and Mesozoic rocks in the north are thrusted over Eocene rocks in the frontal Alborz folds to the south (Berberian et al., 1993). The structural geometry of the fault, together with its high angle of dip, indicates that it is a basement involved thrust (e.g. Mitra and Mount, 1998).

The Mosha fault, has experienced several earthquakes of magnitude greater than 6.5 in the years 958, 1665 and 1830 (Berberian and Yeats, 2001). During the XIX century, no strong events were located near Tehran, but several of magnitude greater than 5 was associated with the Mosha fault (Fig. 1).

The magnetization of titanomagnetite-bearing rocks varies under the mechanical stress and consequent time-dependent local magnetic changes. This phenomenon which is known as piezomagnetic field has been inspired by many investigations in earthquake prediction studies and has been reported in the literature (Johnston and Muller, 1987; Sasai and Ishikawa, 1991; Johnston et al., 1994). Several theories proposed for relating piezomagnetic changes with magnetic signals accompanying earthquakes in the vicinity of a fault. Among all efforts, Sasai (1980) successfully developed what is called Green function method for calculating the piezomagnetic effects due to dislocations. Later, based on his theory, Utsugi et al. (2000), derived an analytical solution for the elementary piezomagnetic potentials due to an inclined rectangular fault within a semi-infinite elastic medium.

Published by Copernicus Publications on behalf of the European Geosciences Union. 


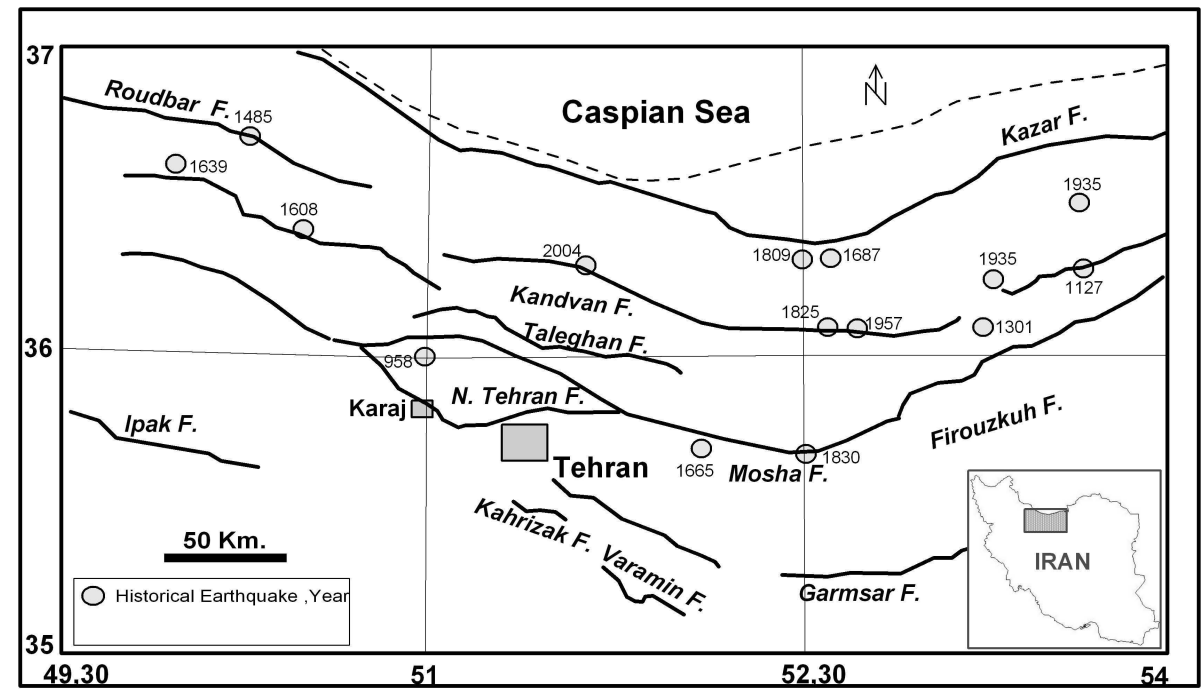

Fig. 1. Main major faults at the study area. Locations of historical earthquake of the Mosha Fault are also shown.

Table 1. The magnetic parameters used for calculation of the rotational piezomagnetic changes for the Mosha fault.

\begin{tabular}{cccc}
\hline $\begin{array}{c}\text { Average } \\
\text { Magnetization }(\mathrm{J}) \\
(\mathrm{A} / \mathrm{m})\end{array}$ & $\begin{array}{c}\text { The main Field at } \\
\text { the fault } \\
\text { location }(\mathrm{nT})\end{array}$ & $\begin{array}{c}\text { Magnetic } \\
\text { Inclination } \\
\text { (degree) }\end{array}$ & $\begin{array}{c}\text { Magnetic } \\
\text { Declination } \\
\text { (degree) }\end{array}$ \\
\hline 1 & 47850 & 54.051 & 4.05 \\
\hline
\end{tabular}

However, the low amplitude nature of piezomagnetic total field changes are imposing serious constrains on our ability for effectively detect this important signals. Mostly they are below the noise level of the measurements. Therefore it seems necessary to use other methods such as measuring rotational piezomagnetic changes in conjunction with total field studies to provide a new insight to the issue.

For this purpose in this study the Piezomagnetic components in the Cartesian coordinate and consequently the rotational piezomagnetic changes for the Mosha fault has been computed. Hence, model calculations have been conducted in order to forecast the rotational piezomagnetic amplitudes in the case of the occurrence of an arbitrary earthquake along the fault.

This information along with the recently acquired magnetic data in the Mosha fault region (Mokhtari et al., 2008) will be used in installing the future magnetic data acquisition system. The system will be part of other sets of instrumentation for earthquake prediction/precursory study in the central Alborz, north of Tehran. In this paper after a brief introduction of the methodology, through numerical modeling of piezomagnetic component for the Mosha fault will be discussed.

\section{Methodology}

The elementary Piezomagnetic potentials and Piezomagnetic changes in components of the ambient magnetic field have been used in order to find the corresponding relations for the increments in initial declination and inclination at the time of earthquake occurrence.

Let $W^{m}$ be the scalar magnetic potential arising from the effect of stress on $J m$ which is a component of initial magnetization $J$. Therefore, $W^{m}$ can be expressed as (Sasai, 1991):

$W^{m}(r)=\iint \Delta u_{k}(\xi) \omega_{k l}^{m}(\xi, r) v_{l}(\xi) d_{\sum}(\xi)$

In which, $\Delta u$ denotes a dislocation vector, and $\omega_{k l}^{m}$ expresses the elementary Piezomagnetic potential produced by the $m^{\text {th }}$ component of initial magnetization of the crustal rock at the fault zone. $v_{l}$ stands for a unit vector normal to the surface element $d_{\sum}$ of the fault. Also $(r)$ represents the location of observation point and $\xi$ denotes the location of the point dislocation source.

Considering $W$ as a scalar potential for the piezomagnetic field of an inclined strike slip fault which has located above the isothermal Curie, a computer program using MATLAB (The MathWorks, Inc.) have been developed. Using this program the Piezomagnetic components in the Cartesian coordinate and consequently the rotational piezomagnetic changes for the above mentioned fault type can be computed. Hence, a model calculation could be carried out in order to forecast the rotational Piezomagnetic amplitudes in the case of the occurrence of an arbitrary earthquake along the fault. The above mentioned methodology has been applied to Mosha fault, which are described in the following section. 
Table 2. The fault parameters used for calculation of the rotational piezomagnetic changes for the Mosha fault.

\begin{tabular}{ccccccccc}
\hline $\begin{array}{c}\text { Fault } \\
\begin{array}{c}\text { Length }(2 \mathrm{~L}) \\
(\mathrm{Km})\end{array}\end{array}$ & $\begin{array}{c}\text { Fault } \\
\text { Width }(\mathrm{W}) \\
(\mathrm{Km})\end{array}$ & $\begin{array}{c}\text { Rigidity } \\
(\mu) \\
(\mathrm{cgs})\end{array}$ & $\begin{array}{c}\text { Poisson's } \\
\text { Ratio } \\
(\nu)\end{array}$ & $\begin{array}{c}\text { Curie } \\
(\mathrm{H}) \\
(\mathrm{Km})\end{array}$ & $\begin{array}{c}\text { Stress } \\
\text { Sensibility } \\
(\beta)\left(\mathrm{bar}^{-1}\right)\end{array}$ & $\begin{array}{c}\text { Dip } \\
(\text { degree })\end{array}$ & $\begin{array}{c}\text { Fault } \\
\text { strike }\end{array}$ & $\begin{array}{c}\text { Depth } \\
(\mathrm{Km})\end{array}$ \\
\hline 70 & 1 & $3.5 \times 10^{11}$ & 0.25 & 15 & $1.0 \times 10^{-4}$ & 75 & E-W & 12 \\
\hline
\end{tabular}
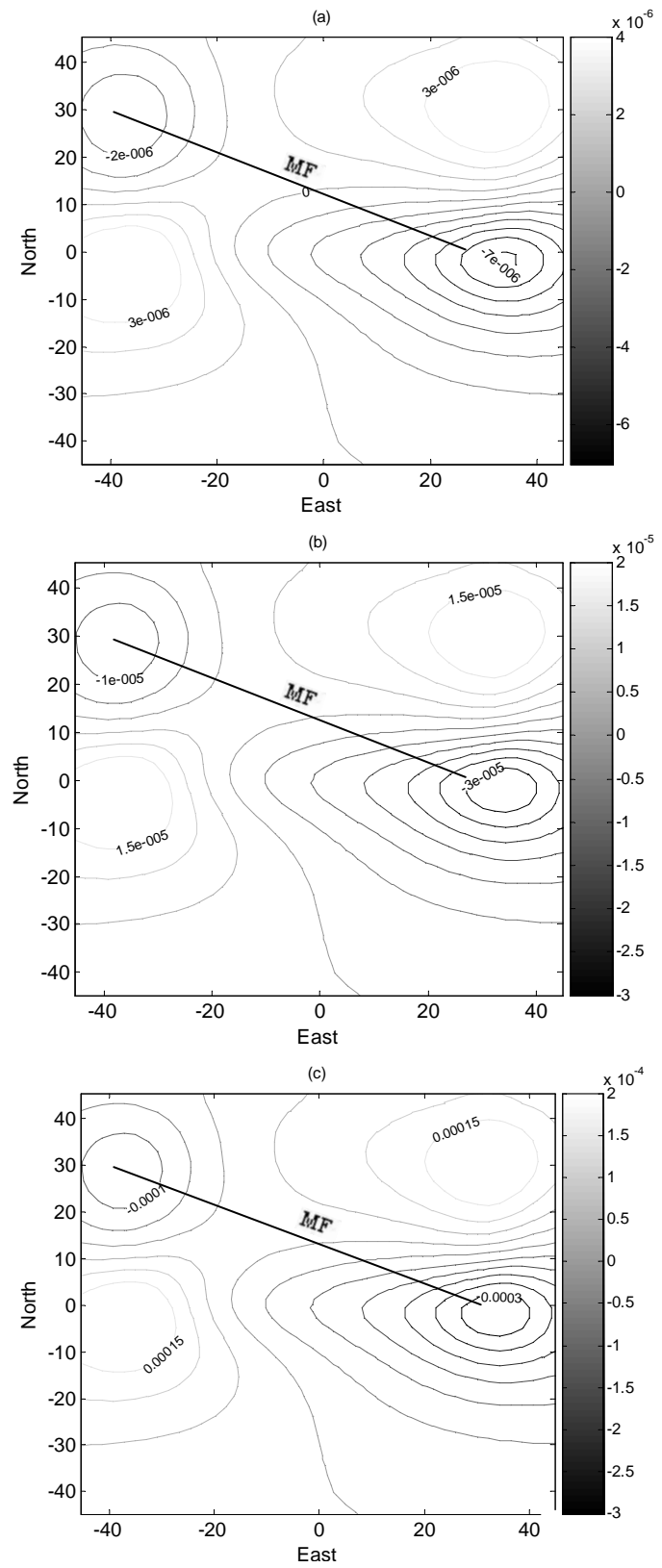

Fig. 2. Declinational Piezomagnetic changes in the case of an amount of $1 \mathrm{~m}$ slip along the Mosha fault (MF), in which the ambient fields are (a) $47850 \mathrm{nT}$ (b) $10000 \mathrm{nT}$ and (c) $1000 \mathrm{nT}$.
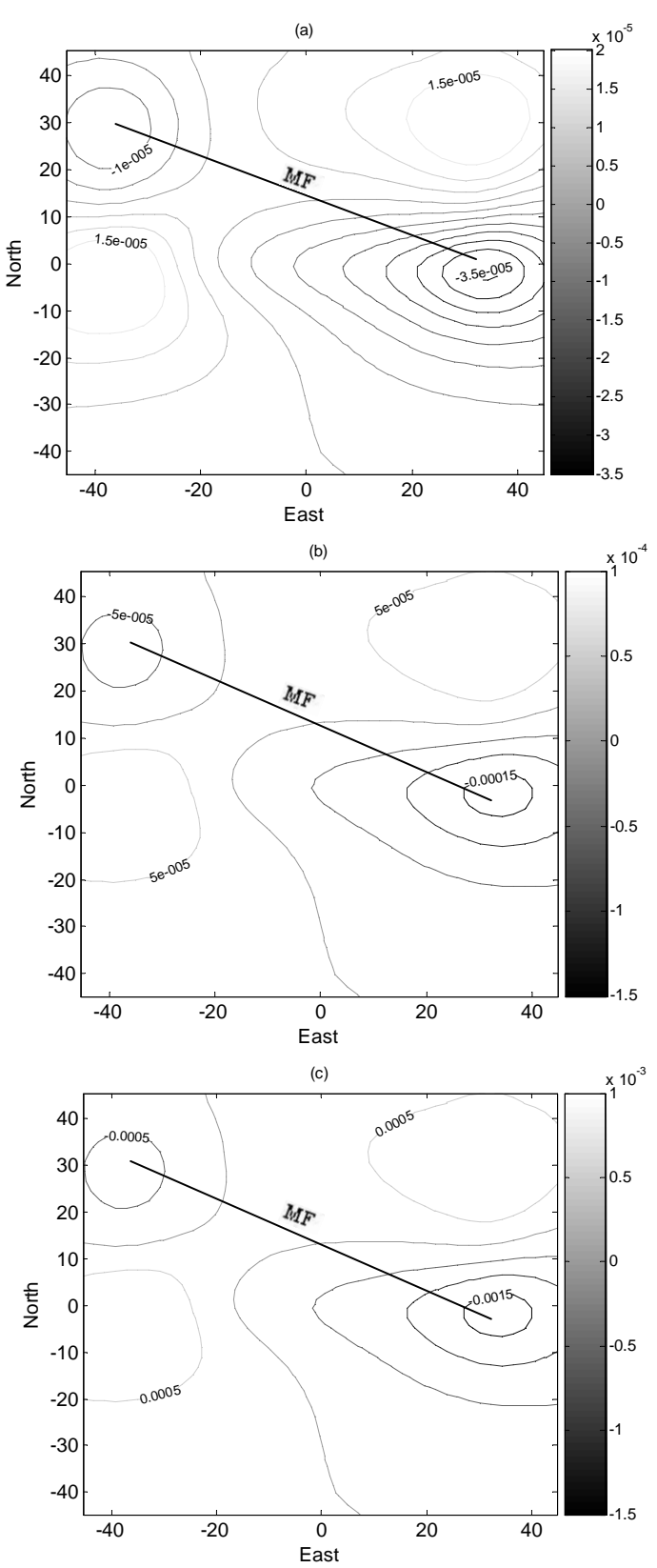

Fig. 3. Declinational Piezomagnetic changes in the case of an amount of $5 \mathrm{~m}$ slip along the Mosha fault (MF), in which the ambient fields are (a) $47850 \mathrm{nT}$ (b) $10000 \mathrm{nT}$ and (c) $1000 \mathrm{nT}$. 

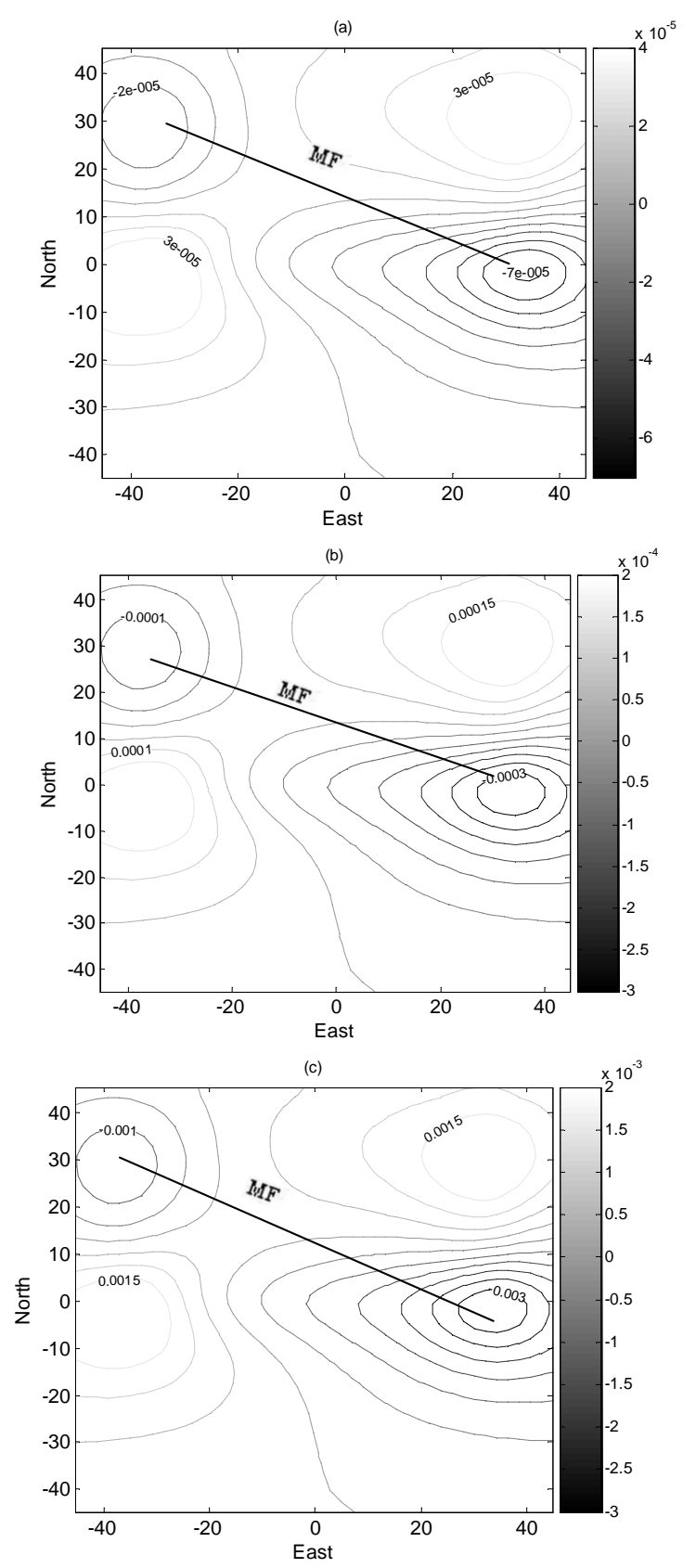

Fig. 4. Declinational Piezomagnetic changes in the case of an amount of $10 \mathrm{~m}$ slip along the Mosha fault (MF), in which the ambient fields are (a) $47850 \mathrm{nT}$ (b) $10000 \mathrm{nT}$ and (c) $1000 \mathrm{nT}$.

\section{Numerical examples}

The Mosha fault as a case study has been selected based on its character as being a major active fault to examine the rotational piezomagnetic changes (both Declination and Inclination). In this regards the order of the rotational piezomagnetic changes for earthquakes with specific magnitudes (due to different amount of slip) have been evaluated at the presence of three different main geomagnetic fields (Table 1). The magnetic parameters are taken from the recent magnetic survey conducted in the Mosha fault region (Mokhtari et al., 2008). The other main parameters of the fault are based on Ashtari et al. (2005), Moinabadi and Yassaghi (2007), Ritz et al. (2006) and Guest et al. (2006) given in Table 2.

Using the computer program developed during this study, both declination and inclination changes along the Mosha fault have been examined. Among all the cases being considered, declinational changes showed more significant anomalous behavior compared to inclinational variations which were nearly zero. Therefore, in the following only declinational changes have been illustrated in all figures and are being discussed.

Figures 2-4 show the declinational piezomagnetic changes in the case of 1,5 and $10 \mathrm{~m}$ slip along the fault, in which the ambient fields are (a) actual value of $47850 \mathrm{nT}$ (b) assumed value of $10000 \mathrm{nT}$ and (c) assumed value of $1000 \mathrm{nT}$ respectively. The assumed values could be modified and the developed computer program has the capacity of handling any values. The above values are only to illustrate the trend or behavior of the piezomagnetic changes under different situations.

The first result that can be inferred from the figures is that the rotational piezomagnetic changes are completely localized at the fault tips. So we may loose these significant data if the instruments being installed even a small distance from these locations. Mokhtari et al. (2007) and Groshkov et al. (2007) using Morphostructural Zoning Method have discussed the most likely occurrence of earthquake events in the Alborz region and showed that these are being associated with intersection of faults which is in agreement with the above mentioned results.

The other important element that affects the piezomagnetic changes is the amount of slip (magnitudes) along the fault. As shown in Figs. 2-4 by an increase in the amount of slip the changes would become more intensified.

In addition the influence of a specific magnetic field at the fault tips has been examined. A careful study of all numerical examples reveals that by decrease in the value of magnetic field from the real amount; cases (a) to assumed value $1000 \mathrm{nT}$; cases (c), the amplitude of changes show a significant increase in the amplitude of the anomalies (Figs. 2-4).

\section{Conclusions}

In this study a computer program under MATLAB (The MathWorks, Inc.) has been developed for computation of the rotational piezomagnetic changes for the Mosha fault. Based on the result it has been concluded that among the parameters examined the amount of slip and the value of total magnetic field has major contribution. In addition due to concentration of changes at the tips of fault, for future observation of the 
piezomagnetic changes for earthquake prediction/precursor purposes it is important to install the magnetometers very close to these locations.

The above mentioned result will be further examined along with other earthquake precursory methods in Central Alborz, north of Tehran.

Edited by: P. F. Biagi

Reviewed by: two anonymous referees

\section{References}

Ashtari, M., Hatzfeld, D., and Kamalian, N.: Microseismicity in the region of Tehran, Tectonophysics, 395, 193-208, 2005.

Berberian, M., Qorashi, M., Argang Ravesh, B., and Mohajer Ashjaie, A.: Seismotectonics and earthquake-fault hazard investigation in the Tehran Region, contribution to the seismotectonics of Iran - Part 5, Report No 56, Geological Survey of Iran, 1993.

Berberian, M. and Yeats, R. S.: Contribution of archaeological data to studies of earthquake history in the Iranian plateau, J. Struct. Geol., 23, 563-584, 2001.

Dellenbach, J.: Contribution l'e'tude ge'ologique de la re'gion situe'e l'est de Te'he'ran (Iran), Faculty of Science, University of Strasbourg (France), 117 pp., 1964.

Guest, B., Stockli, D. F., Grove, M., Axen, G. J., Lam, P. S., and Hassanzadeh, J.: Thermal histories from the central Alborz Mountains, northern Iran: Implications for the spatial and temporal distribution of deformation in northern Iran, GSA Bulletin, 118, 11/12, 1507-1521, 2006.

Gorshkov, A. Mokhtari, M., Piotrovskaya, E., and Shahpasanzadeh, M.: Identification of Earthqake Prone areas in the Alborz Region, 5th International Conference on Seismology and Earthquake Engineering, Section EP, Tehran, Iran, 13-16 May 2007.
Johnston, M. J. S. and Muller, R. J. : Seismomagnetic observation during the 8 July 1986 magnitude 5.9 North Palm Springs earthquake, Science, 237, 1201-1203, 1987.

Johnston, M. J. S., Muller, R. J., and Sasai, Y.: Magnetic field observations in the near-field of the 28 June 1992, Mw 7.3 Landers., California, earthquake, Bull. Seism. Am., 84, 792-798, 1994.

Mitra, S. and Mount, V. S.: Foreland basement involved structures, Am. Assoc. Petr. Geol. B., 82(1), 70-109, 1998.

Moinabadi, M. E. and Yassaghi, A.: Geometry and kinematics of the Mosha Fault, south central Alborz Range, Iran: An example of basement involved thrusting, J. Asian Earth Sci., 29, 928-938, 2007.

Mokhtari, M., Groshkov, A., and Shahpasandzadeh, M.: Identification of Seismic pattern in the Central Alborz using morphostructural zone method for earthquake of larger or equal 6 , Internal technical publication of IIEES, 145 pp., 2007.

Mokhtari, M., Ovisi Moakhr, M., Asgari, A. H., and Shah Nazari, H.: Acquisition of Magnetic data North of Tehran (Mosha Fault Region), IIEES internal report (in Farsi), 48 pp., 2008.

Ritz, J.-F., Nazari, H., Ghassemi, A., Salamati, R., Shafei, A., Solaymani, S., and Vernant, P.: Active transtension inside central Alborz: A new insight into northern Iran - southern Caspian geodynamics, Geology, 34(6), 477-480, 2006.

Sasai, Y.: Application of the elasticity theory of dislocations to tectonomagnetic modeling, Bull, Earthq. Res. Inst., Univ. Tokyo, 55, 387-447, 1980.

Sasai, Y.: Tectonomagnetic modeling on the basis of the linear Piezomagnetic effects, Bull Earthq. , 66, 585-722, 1991.

Sasai, Y. and Ishikawa, Y.: Tectonomagnetic signals related to the seismomagnetic activity in the Izu Peninsula, J. Phys. Earth, 39, 299-319, 1991.

Utsugi, M., Nishida, Y., and Sasai, Y.: Piezomagnetic potentials due to an inclined rectangular fault in a semi-infinite medium, Geophys. J. Int., 140, 479-492, 2000. 